\begin{tabular}{lllllll}
\multicolumn{6}{l}{ Abstract IDDF2021-ABS-0064 Table 1 } \\
\hline & & N & Mean & S.D & F-value & p-value \\
\hline AF total & NE & 32 & 289.4 & 101.1 & 164.685 & $0.0005^{* *}$ \\
cell & RE & 174 & 55.7 & 60.6 & & \\
count & SBP & 42 & 527.6 & 272.2 & & \\
\hline
\end{tabular}

${ }^{* *}$ Significance at $p<0.01$

\begin{tabular}{|c|c|c|c|c|c|c|}
\hline \multicolumn{7}{|c|}{ Abstract IDDF2021-ABS-0064 Table 2} \\
\hline & & $\mathrm{N}$ & Mean & S.D & F-value & p-value \\
\hline Ascitic PMN & $\mathrm{NE}$ & 32 & 181.9 & 25.4 & 212.507 & $0.0005^{* *}$ \\
\hline \multirow[t]{2}{*}{ cell count } & $\mathrm{RE}$ & 174 & 27.2 & 26.5 & & \\
\hline & SBP & 42 & 396.0 & 203.9 & & \\
\hline
\end{tabular}

** Significance at $p<0.01$

\begin{tabular}{|c|c|c|c|}
\hline \multicolumn{4}{|c|}{ Abstract IDDF2021-ABS-0064 Table 3} \\
\hline & $\mathrm{NE}$ & $\mathrm{RE}$ & SBP \\
\hline 1st Month & $6.3 \%$ & $1.7 \%$ & $4.8 \%$ \\
\hline 6th Month & $21.9 \%$ & $8.0 \%$ & $23.8 \%$ \\
\hline
\end{tabular}

Results 248 patients were included,174-RE,42-SBP,32-NE. There was a statistically significant inter-group difference in mean total leukocyte count, blood urea, sodium, bilirubin, and AF total cell count and AF PMN cell count. There was no statistically significant difference with CTP, MELD-Na, AF albumin, Protein and creatinine, S.albumin, PT/INR. The mean AF total cell count - IDDF2021-ABS-0064 Table 1 and AF PMN - IDDF2021-ABS-0064 Table 2, Outcome- IDDF2021ABS-0064 Table 3.

Outcome -The mortality in NE was more at first month and comparable to SBP at sixth month as in IDDF2021-ABS0064 Table 3.

Conclusions AF PMN cell count has considerable prognostic value in patients with cirrhosis and is independent of AF protein, albumin, MELD-Na, CTP, creatinine. Mortality rate was comparable in NE and SBP. This emphasizes the need for early detection and aggressive management of patients with AF PMN cell count $>150$ cells $/ \mathrm{mm}^{3}$, lowering the threshold for antibiotic initiation.

\section{IDDF2021-ABS-0067 THE COMPARISON BETWEEN OVERALL SURVIVAL OF TRANSARTERIAL CHEMOEMBOLIZATION AND BEST SUPPORTIVE CARE IN INTERMEDIATE- STAGE HEPATOCELLULAR CARCINOMA}

\footnotetext{
${ }^{1}$ Keerati Akarapatima*, ${ }^{1}$ Arunchai Chang, ${ }^{2}$ Tanaporn Prateepchaiboon, ${ }^{2}$ Nuttanit Pungpipattrakul, ${ }^{3}$ Apiradee Songjamrat, ${ }^{3}$ Songklod Pakdeejit, ${ }^{1}$ Attapon Rattanasupar. 'Division of Gastroenterology, Department of Internal Medicine, Hatyai Hospital, Songkhla, Thailand; '2Department of Internal Medicine, Hatyai Hospital, Songkhla, Thailand; ${ }^{3}$ Division of Intervention Radiology, Department of Radiology, Hatyai Hospital, Songkhla, Thailand
}

Background The newly European Association for the Study of the Liver (EASL) suggests performing transarterial chemoembolization (TACE) for only intermediate stage HCC with compensated cirrhosis (Child-Pugh score 5-6). However, The Thai HCC guideline 2021 allows both compensated cirrhosis and early decompensated cirrhosis (Child-Pugh score 7-8) intermediate stage HCC for TACE. Therefore, our study aims to compare overall survival (OS) between TACE and best supportive care (BSC) of Child-Pugh score 5-8 cirrhosis HCC patients and subgroup analysis in compensated cirrhosis and decompensated cirrhosis.

Methods We retrospectively assessed 118 intermediate-stage HCC patients. Comparison of OS between TACE and BSC were calculated using the Kaplan-Meier method. In addition, the Cox proportional-hazards model was used to evaluate survival-predictive variables and the relationship between the TACE and OS.

Results The 118 intermediate-stage HCC patients were included. There were $32(27.1 \%)$ female patients. The mean age was $59.0 \pm 11.4$ years, and the mean body mass index was $23.2 \pm 4.5(\mathrm{~kg} / \mathrm{m} 2)$. There were $50(42.4 \%)$ hepatitis B infected patients, 28 (23.7\%) hepatitis C infected patients, and $33(28.0 \%)$ alcohol-related liver disease patients. The compensated cirrhosis was 79 (66.9\%) patients. Among all patients, HCC patients who underwent TACE had increased median OS comparing to those who

Compensated and early decompensated cirrhosis

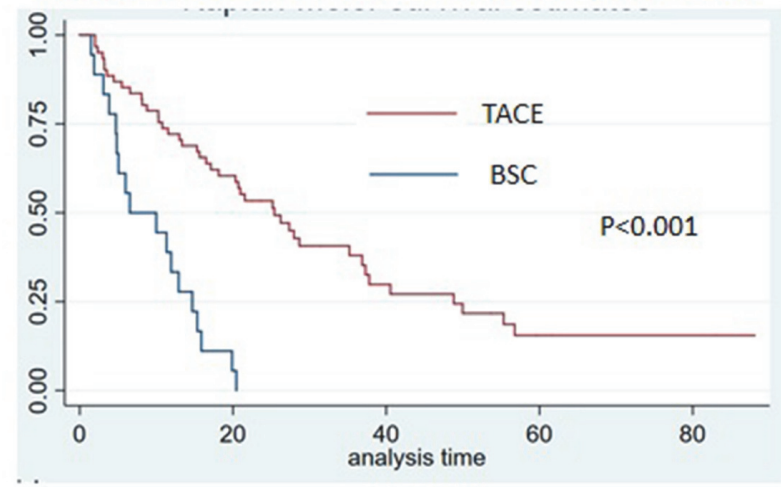

Abstract IDDF2021-ABS-0067 Figure 1

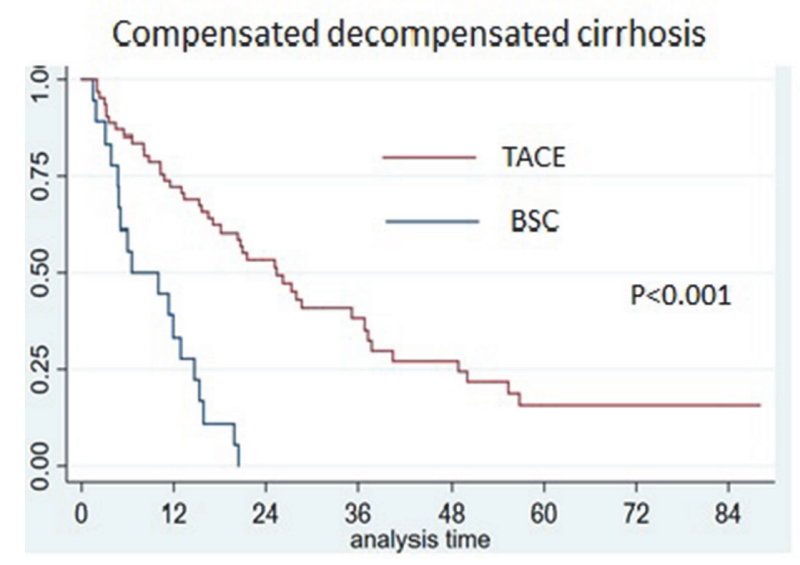

Abstract IDDF2021-ABS-0067 Figure 2 


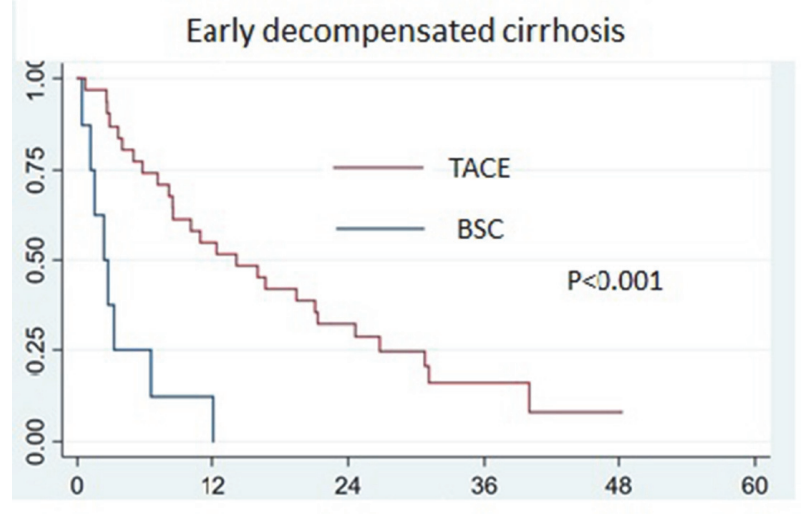

Abstract IDDF2021-ABS-0067 Figure 3

received BSC (21.4 VS 8.2 months, Log-rank $\mathrm{P}<0.001)$. Subgroup analyses showed patients undergoing TACE had increased median OS comparing to those who received BSC in both compensated cirrhosis (26.0 VS 9.0 months, Log-rank $\mathrm{P}<0.001)$ and early decompensated cirrhosis (14.5 VS 6.9 months, Log-rank $\mathrm{P}<0.001)$. In the Cox proportional-hazards model, undergoing TACE is an independent factor associated with prolonged OS in overall patients (Hazard ratio; HR 0.29, 95\% CI 0.17-0.49, $\mathrm{P}<0.001$ ), compensated cirrhosis (HR 0.31, 95\% CI 0.16-0.62, $\mathrm{P}<$ 0.001 ), and early decompensated cirrhosis (HR 0.16, 95\% CI 0.061-0.44, $\mathrm{P}<0.001)$.

(IDDF2021-ABS-0067 Figure 1, IDDF2021-ABS-0067 Figure 2, IDDF2021-ABS-0067 Figure 3)

Conclusions This study demonstrated the beneficial effect of TACE on OS in patients with intermediate-stage HCC who had compensated and early decompensated cirrhosis.

\section{IDDF2021-ABS-0070 PREDICTIVE PERFORMANCE OF THE HAP SCORE AT PATIENTS WITH HEPATOCELLULAR CARCINOMA FOLLOWING TRANS-ARTERIAL CHEMOEMBOLISATION (TACE)}

Anh Tran Ngoc*, Hieu Nguyen Trung, Lieu Dau Quang. Hanoi Medical University Hospital, Vietnam

\subsection{6/gutjnl-2021-IDDF.77}

Background Transarterial chemoembolization (TACE) is the therapy which have chosen in $50-60 \%$ of patients with Hepatocellular carcinoma (HCC). Recently, Hepatoma Arterial Embolization (HAP) score stratifies patients who will benefit from the first TACE. We conducted a survey to investigate the value of HAP score in the prognosis of HCC treated with TACE in Vietnam.

Methods Prospective cohort study in patients with HCC and first TACE at the Department of Internal Medicine, Hanoi Medical University Hospital, Vietnam, from January 2017 to June 2021. The HAP score (serum albumin $0.9 \mathrm{mg} / \mathrm{dl}$, alphafoetoprotein $>400 \mathrm{ng} / \mathrm{ml}$, and tumor size $>7 \mathrm{~cm}$ ), and ALBI score was applied before first TACE, mortality and survival were observed with a follow-up of 54 months.
Results We included 106 patients with age of $62.8 \pm 10.1$ years. In this population, HCC risk factor was mainly hepatitis B with 75.5\% (80/106). The HAP score classified 19, 41, 31 and 15 patients as HAP A, B, C and D, respectively,

Overall Comparisons

\begin{tabular}{|l|r|r|c|}
\hline & Chi-Square & df & $p$ \\
\hline Log Rank (Mantel-Cox) & 17.863 & 3 & $<0.001$ \\
\hline
\end{tabular}

Test of equality of survival distributions for the different levels of HAP.

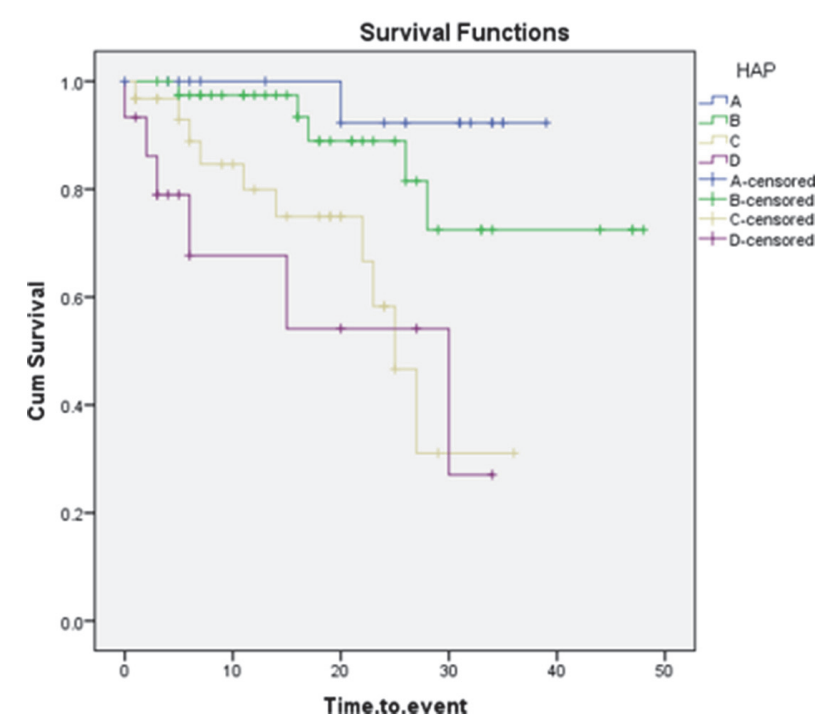

Abstract IDDF2021-ABS-0070 Figure 2 Kaplan-Meier curve for HAP score

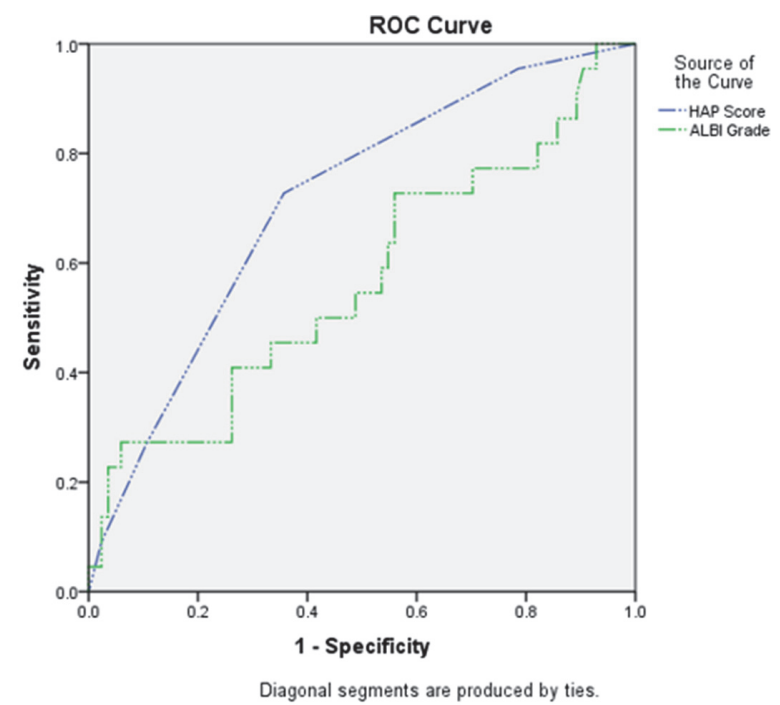

Area Under the Curve

\begin{tabular}{|l|c|}
\hline Test Result Variable(s) & Area \\
\hline HAP Score & .711 \\
ALBI.Diem & .568 \\
\hline
\end{tabular}

Abstract IDDF2021-ABS-0070 Figure 3 ROC curves for HAP score and ALBI grade 